\title{
Sevoflurane ameliorates doxorubicin-induced myocardial injury by affecting the phosphorylation states of proteins in PI3K/Akt/mTOR signaling pathway
}

\author{
Yini $\mathrm{Wu}^{1}$, Jianping Wang ${ }^{2}$, Xiaoyan $\mathrm{Yu}^{1}$, Dongli $\mathrm{Li}^{1}$, Xin $\mathrm{Han}^{1}$, Lihua $\mathrm{Fan}^{1}$ \\ ${ }^{1}$ Department of Anesthesiology, Wenzhou Medical College, the Sixth Affiliated Hospital, Lishui, China \\ ${ }^{2}$ Department of General Surgery, Wenzhou Medical College, the Fifth Affiliated Hospital, Lishui, China
}

\begin{abstract}
Background: The effect of sevoflurane on the doxorubicin-induced myocardial injury was explored by investigating the phosphorylation states of proteins in phosphatidylinositol 3-kinase (PI3K)/Akt/mammalian target of rapamycin ( $m$ TOR) signaling pathway.

Methods: Myocardial injury rat models were induced by doxorubicin and evenly assigned into five groups according to different treatment: Doxorubicin group (DG, 200- $\mu$ L saline solution), sevoflurane group (SevG, inhaled with 2.4\% sevoflurane for $2 \mathrm{~h}$ ), LY294002 group ( $L Y G$, Akt inhibitor, $0.3 \mathrm{mg} / \mathrm{kg}$ in 200- $\mu$ L Dimethyl Sulfoxide [DMSO]), solvent DMSO control group (SG) and autophagy inhibitor 3-methyladenine (3-MA) group (MG, $30 \mathrm{mg} / \mathrm{kg}$ in 200- $\mu \mathrm{L}$ DMSO). The healthy rats were assigned to

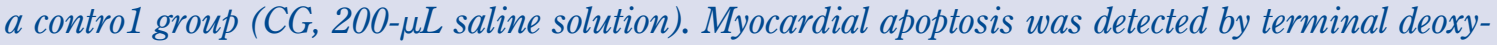
nucleotidyl transferase dUTP nick end labeling (TUNEL) assay. The concentration of cardiac troponin I (cTnI) was detected by ELISA. The levels of total Akt (t-Akt), phosphorylated Akt ( $p$-Akt), mammalian target of rapamycin ( $m$ TOR), phosphorylated-mTOR ( $p$-mTOR) and autophagy marker LC3-II was detected by Western Blot. The experiments were also repeated at the cell level.

Results: Terminal deoxynucleotidyl transferase dUTP nick end labeling analysis showed that the apoptosis rates were high in $D G$ and $S G$, reached the highest level in $L Y G$, reduced in SevG and $M G$, and reached the lowest level in $C G$. The levels of p-Akt p-mTOR were low in groups $D G$ and $S G$, reached the lowest level in $L Y G$, increased in SevG and $M G$, and reached the highest level in CG. In contrast, LC3-II expression, apoptosis index and serum cTnI concentration were high in DG and SG, reached the highest level in $L Y G$, reduced in SevG and $M G$, and reached the lowest level in $C G(p<0.05)$. Cell experiment showed similar results as with animal experiments.

Conclusions: Sevoflurane ameliorates myocardial injury by affecting the phosphorylation states of the proteins in PI3K/Akt/mTOR signaling pathway and reducing the injury biomarker. (Cardiol J 2017; 24, 4: 409-418)
\end{abstract}

Key words: cardiac injury, doxorubicin, phosphatidylinositol 3-kinase, phosphorylated rapamycin, protein-serine-threonine kinases

\section{Introduction}

Chronic heart failure is a serious threat to public health and can be caused by many heart disorders, such as myocardial infarction, chronic hypertension and toxic agents. The anthracycline doxorubicin (DOX) is widely used for tumor therapy, but its wide use often leads to heart failure [1].

Address for correspondence: Dr. Lihua Fan, Department of Anesthesiology, Wenzhou Medical College, the Sixth Affiliated, Hospital, No.15, Dazhong Street, Lishui 323000, Zhejiang Province, China, tel: +86 5782780072, e-mail: fanlihua19@126.com Received: 12.09.2016 Accepted: 23.11.2016 
Many approaches are implemented to protect the heart against DOX-induced cardiac injury, but the problems remain unresolved.

Anesthetic sevoflurane has been widely used clinically and it has the lowest solubility, allowing rapid induction and recovery. Sevoflurane shows better protective functions for cardiac injury. On the other hand, phosphatidylinositol 3-kinase (PI3K) has been reported to be involved in myocardial function, such as contractility. A previous study showed that myocardial dysfunction was controlled when phosphatidylinositol 3-kinase (PI3K) was inhibited in the rat model with a decrease in the phosphorylation of myocardial phospholamban [2]. Serine/ /threonine protein kinase Akt can be activated by various extracellular stimuli in a PI3K-dependent manner and regulates many functions such as cardiac survival, growth and metabolism. Akt signaling pathway plays an important role in cardiac growth, contractile function, and coronary angiogenesis [3]. A previous study demonstrated that sevoflurane reduced DOX-induced rat cardiac injury [4]. Thus, sevoflurane improves DOX-induced cardiac injury perhaps by affecting Akt signaling pathway.

Doxorubicin can induce cardiomyocytes apoptosis and shows obvious cardiac toxicity [5]. DOX-induced myocardial toxicity which may involved in many complications, such as energy metabolism, free radical injury, calcium overload and apoptosis factors, all of which result in myocardial injury. Furthermore, DOX has been found to be associated with the changes of cellular autophagy [6], which is also involved in the pathogenesis of myocardial injury [7]. Light chain 3 (LC3)-II [8], mTOR [8] and Akt [8] are important autophagy markers. It has been confirmed that sevoflurane reduces myocardial injury by inhibiting autophagy and activating PI3K/Akt signaling pathway [9]. Furthermore, phosphorylation at Anti-p (Ser473) of Akt, along with Thr308 of its activation loop, is critical for Akt function. Akt-Ser473 phosphorylation is required for rictor-mTOR (TORC2) [10]. A previous study showed that Akt phosphorylation inhibited the apoptosis of endothelial cells. Activation of the serine/threonine kinase Akt contributed to the changes in endothelial morphology and function [11]. Phosphorylated-Akt has been shown to be associated with phosphorylated-mTOR in most ovarian tumors with an evaluated phosphorylation level [12]. Results suggest that the phosphorylation states of proteins are important for the activity of PI3K/Akt/ /mTOR signaling pathway. Cardiac troponin I (cTnI), a biomarker for the diagnosis of acute myocardial infarction, is also associated with cardiac injury [13]. Therefore, it was hypothesized that sevoflurane treatment ameliorates cardiac injury and may affect the phosphorylation states of proteins of PI3K/Akt/ /mTOR pathway and related important molecules.

\section{Methods}

Myocardial cells H9c2 were purchased from Cell Bank of Shanghai Institute of Cell Biology, Chinese Academy of Sciences (Shanghai, China). A total of 36 Sprague-Dawley rats (8 weeks, weight 200-250 g) were purchased from the Animal Center of Wenzhou Medical University (Wenzhou, China). Anti-p-Akt (Ser473) antibody (4060), Anti-p-mTOR antibody (5536) and Anti-mTOR antibody (2972) were purchased from Cell Signaling Company (Danvers, MA, USA). Anti-LC3 antibody (L7543), anti-glyceraldehyde 3-phosphate dehydrogenase (GAPDH) antibody, LY294002 (a potent inhibitor of numerous proteins with morphine-containing chemical compound) and 3-methyladenine (3-MA) were purchased from Sigma (St. Louis, MO, USA).

\section{Establishment of myocardial injury model}

Before the experiment, all protocols were approved by the Animal Ethical Committee of Wenzhou Medical College (Wenzhou, China). According to a previous report [14], 30 rats were intraperitoneally injected with DOX at $4 \mathrm{mg} / \mathrm{kg}$ in $200-\mu \mathrm{L}$ saline solution weekly for 6 weeks. Meanwhile, the 6 healthy rats were intraperitoneally injected with $200-\mu \mathrm{L}$ saline solution as a control group. Myocardial injury model was confirmed by rat growth, the ratio of heart weight and body weight, electrocardiograms (ECGs) analysis and the TUNE analysis for myocardial tissues. The cardiac marker lactate dehydrogenase (LDH) was measured by using Lactate Dehydrogenase Assay Kit from Sigma (MAK066). Creatine kinase-MB (CK-MB) was detected by using Creatine Kinase Activity Assay Kit from Sigma (MAK116).

\section{Grouping}

Myocardial injury rat models were induced by DOX and randomly divided into five groups according to different treatment: Doxorubicin group (DG, $200-\mu \mathrm{L}$ saline solution), sevoflurane group (SevG), LY294002 group (Akt inhibitor, LYG), Solvent DMSO group (SG) and autophagy inhibitor 3-methyladenine (3-MA) group (MG). Meanwhile, the healthy rats were assigned as a control group (CG, 200- $\mu \mathrm{L}$ saline solution) (Fig. 1). In groups $C G$ and $D G$, the rats were mechanically ventilated for $2 \mathrm{~h}$. In SevG, the rats were inhaled with $2.4 \%$ sevoflurane for $2 \mathrm{~h}$. In LYG, the rats 


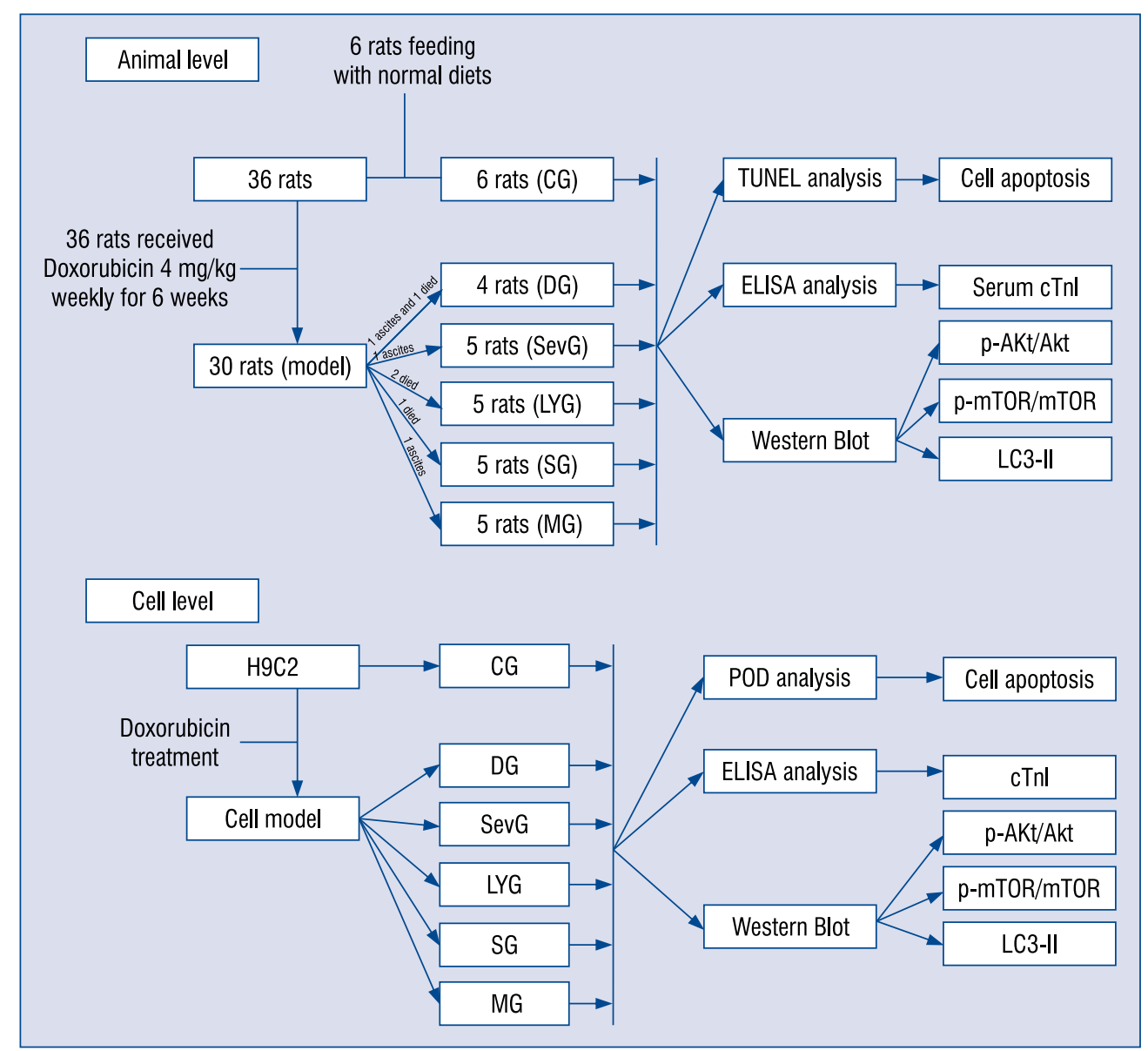

Figure 1. The flowchart of present study. Myocardial injury rat models were induced by doxorubicin and randomly divided into five groups according to different treatments: Doxorubicin group (DG), sevoflurane group (SevG), LY294002 group (Akt inhibitor, LYG), Solvent DMSO control group (SG) and autophagy inhibitor 3-methyladenine (3-MA) group (MG). Meanwhile, the healthy rats were assigned as a control group (CG). In SevG, the rats were inhaled with $2.4 \%$ sevoflurane for $2 \mathrm{~h}$. In LYG, the rats were intraperitoneally injected with LY294002 $0.3 \mathrm{mg} / \mathrm{kg}$ injection in $200-\mu$ L DMSO. In SG, the rats were intraperitoneally injected with the equal volume of DMSO. In MG, the rats were intraperitoneally injected with 3-MA at $30 \mathrm{mg} / \mathrm{kg}$.

were intraperitoneally injected with LY294002 $0.3 \mathrm{mg} / \mathrm{kg}$ injection in $200-\mu \mathrm{L}$ Dimethyl Sulfoxide (DMSO). In SG, the rats were intraperitoneally injected with $200-\mu \mathrm{L}$ DMSO. In MG, the rats were intraperitoneally injected with $30 \mathrm{mg} / \mathrm{kg} 3-\mathrm{MA}$ in $200-\mu$ L DMSO.

\section{Cell experiments}

The myocardial cells H9C2 were cultured in high glucose Dulbecco's Modified Eagle's Medium (DMEM) containing 10\% fetal bovine serum at $37^{\circ}$ in $5 \% \mathrm{CO}_{2}$ incubator. Cardiomyocytes were adjusted to $10^{5} / \mathrm{mL}$ and seeded in 6 -well ( $2 \mathrm{~mL} /$ well) culture plate. Doxorubicin was added to each cell at a final concentration of $2 \mu \mathrm{M}$ and treated for $2 \mathrm{~h}$. With the animal experiment, all the cells were assigned to six groups ( $\mathrm{n}=6$ for each group) accord- ing to different treatment: CG, DG, SevG, LYG, SG and MG) (Fig. 1). In CG, the cells were not treated by DOX. In DG, $1 \mathrm{~mL}$ cells were incubated with $4 \mu \mathrm{g} / \mathrm{mL}$ for $2 \mathrm{~h}$. In LYG, $1 \mathrm{~mL}$ cells were treated LY294002 with a final concentration of $20 \mu \mathrm{M}$ in $1-\mu \mathrm{L}$ DMSO. In SevG, the cells were treated $2.4 \%$ sevoflurane for $2 \mathrm{~h}$. In SG, $1 \mathrm{~mL}$ cells were treated with $1-\mu \mathrm{L}$ DMSO for $2 \mathrm{~h}$. In MG, $1 \mathrm{~mL}$ cells were treated with 3-MA at a final concentration of $5 \mathrm{mM}$ in $1-\mu \mathrm{L}$ DMSO. All the following experiments were performed at cellular level.

\section{TUNEL analysis for cardiac} myocyte apoptosis

Apoptotic cells were identified by the TUNEL assay and quantitated under a microscope by a single observer. Cell number was counted under 
Table 1. The comparison of body weight and heart weight between healthy rats and model rats after 6 -week doxorubicin treatment.

\begin{tabular}{lccc}
\hline Groups & Body weight $[\mathrm{g}]$ & Heart weight $[\mathrm{g}]$ & Heart weight/Body weight $[\mathrm{mg} / \mathrm{g}]$ \\
\hline CG & $238.12 \pm 29.25$ & $0.75 \pm 0.07$ & $2.96 \pm 0.34$ \\
DG & $199.37 \pm 28.48^{*}$ & $0.76 \pm 0.09$ & $3.68 \pm 0.46^{*}$ \\
\hline
\end{tabular}

${ }^{*} p<0.05$ via a CG group; CG - healthy rats; DG — doxorubicin-induced myocardial injury rats

a high-power from six different views. Promyelocytic leukemia oncogenic domain (POD) apoptosis detection kit (Roche, USA) was used to detect apoptotic cells.

\section{Enzyme-linked immunosorbent assay (ELISA) analysis}

Cardiac troponin I concentration were analyzed in myocardial tissue or cultured cells by using an Rat Cardiac Troponin I ELISA Kit Life from Diagnostics, Inc. (Cat. No. CTNI-2-HS, West Chester, PA, USA).

\section{Western Blot analysis}

The myocardial tissue $(500 \mathrm{mg})$ was excised from the experimental rats and the protein levels of P-Akt, Akt, P-mTOR, mTOR and LC3-II were analyzed by Western Blot. Myocardial tissue was grounded with a pellet pestle rinder (Kontes Glass, Vineland, NJ, USA) for $3 \mathrm{~min}$. The isolated cells were collected by rapid filtration onto glass fiber filters. The cells were treated with a proteinase inhibitor cocktail, $2 \mathrm{mM} \mathrm{N}$-ethylmaleimide, $2 \mathrm{mg} / \mathrm{mL}$ aprotinin, $4 \mathrm{mg} / \mathrm{mL}$ pepstatin, ten $\mathrm{mg} / \mathrm{mL}$ leupeptin, and $2 \mathrm{mM}$ phenylmethylsulphonyl fluoride, and lysed by 3 -cycle freezing ( $10 \mathrm{~min}$ ) and thawing (5 min). The supernatant samples (or supernatants of cultured cells) were collected by centrifuge and solubilized in the buffer with $50 \mathrm{mM}$ Tris $\mathrm{HCl}, 10 \%$ glycerol, $1 \%$ SDS, $0.05 \%$ bromophenol blue, and $5 \% \beta$-mercaptoethanol and the $\mathrm{pH}$ was adjusted to $6.8 .10-\mu \mathrm{g}$ protein from each cell lysate were loaded onto $10 \%$ sodium dedecyl sulfate polyacrylamide gel electrophoresis and electrophoresed at $200 \mathrm{~V}$ for $2 \mathrm{~h}$. After electrophoresis, the separated proteins were transferred to a polyvinylidene fluoride (PVDF) membrane in transfer buffer ( $48 \mathrm{mmol} / \mathrm{L}$ Tris, $39 \mathrm{mmol} / \mathrm{L}$ glycine, $20 \%$ methanol, $\mathrm{pH}$ 9.2) for $2 \mathrm{~h}$ at a constant current of $20 \mathrm{amps}$ and blocked in Tris buffered saline containing $2.5 \%$ non-fat dry milk for $30 \mathrm{~min}$. The membranes were incubated with the antibodies (diluted 1:1000) to P-Akt, Akt, P-mTOR, mTOR and LC3-II overnight at $4^{\circ} \mathrm{C}$. An avidin-biotin complex (ABC) peroxidase and substrate kit was used to detect the primary antibodies bound to the antigen. With X-ray film exposure and photograph, the expression of P-Akt, Akt, P-mTOR, mTOR and LC3-II was measured using Quantity One software with the internal control of $\beta$-actin.

\section{Statistical analysis}

Student's t test was used to compare the levels of weight loss, LDH, CK-MB, P-Akt, Akt, P-mTOR, mTOR and LC3-II between 2 different samples (via a CG group or a DG group). Statistical analysis was conducted in the in vitro experiments by using the independent two-tailed t-test. Statistical analysis was performed with the SPSS 20.0.The p value was regarded as significant if it was less than 0.05 .

\section{Results}

\section{Evaluation of myocardial injury model}

During the experiment, healthy rat weight was gained and there were no deaths. In contrast, model rats reduced their activities resulting in reduced weight, 3 rats had ascites (each one from DG, SevG and MG, respectively) and 4 rats died (1 from DG, 2 from LYG and 1 from SG). The number was too low to be analyzed by using a statistical method. These rats were removed from the final statistical analysis. Thus, there were $6,4,5,4,5,6$ cases in CG, DG, SevG, LYG, SG and MG groups finally, respectively. The mean body weight of model animals was significantly reduced in all models when compared with healthy ones $(\mathrm{p}<0.05$, Table 1$)$. In contrast, the heart weight to body weight were increased significantly in model animals when compared with healthy controls $(p<0.05$, Table 1). Comparatively, the activities of LDH and CK-MB were significantly increased in all models when compared with the healthy animals $(\mathrm{p}<0.05$, Table 2). ECG analysis also showed that model rats had significant ST-segment elevation when compared to controls $(\mathrm{p}<0.05)$. All results suggest the myocardial injury model was successfully established. 
Table 2. The effect of sevoflurane on creatine kinase-MB and lactate dehydrogenase (LDH) activities in doxorubicin-induced myocardial injury models.

\begin{tabular}{lcc}
\hline Groups & CK-MB [IU/L] & LDH [IU/L] \\
\hline CG & $9124.73 \pm 1367.16^{\#}$ & $1955.26 \pm 178.36^{\#}$ \\
DG & $24652.55 \pm 2671.65^{*}$ & $3638.23 \pm 216.45^{*}$ \\
SevG & $16248.31 \pm 2281.49^{*, \#}$ & $2578.51 \pm 198.32^{*, \#}$ \\
LYG & $28954.35 \pm 2956.74^{*, \#}$ & $3942.53 \pm 248.37^{*, \#}$ \\
SG & $25002.71 \pm 2544.23^{*}$ & $3679.39 \pm 224.16^{*}$ \\
MG & $12169.23 \pm 1865.44^{*, \#}$ & $2219.47 \pm 196.53^{*, \#}$ \\
\hline
\end{tabular}

Myocardial injury rat models were induced by doxorubicin and randomly divided into five groups according to different treatments: Doxorubicin group (DG), sevoflurane group (SevG), LY294002 group (Akt inhibitor, LYG), Solvent DMSO control group (SG) and autophagy inhibitor 3-methyladenine (3-MA) group (MG). Meanwhile, the healthy rats were assigned as a control group (CG). In SevG, the rats were inhaled with $2.4 \%$ sevoflurane for $2 \mathrm{~h}$. In LYG, the rats were intraperitoneally injected with LY294002 $0.3 \mathrm{mg} / \mathrm{kg}$ injection in $200-\mu \mathrm{L}$ DMSO. In SG, the rats were intraperitoneally injected with $200-\mu \mathrm{L}$ DMSO. In MG, the rats were intraperitoneally injected with $30 \mathrm{mg} / \mathrm{kg} 3-\mathrm{MA}$ in $200-\mu \mathrm{L}$ DMSO.*p $<0.05$ via a CG group and \#p $<0.05$ via a DG group

\section{Sevoflurane reduced the activities} of LDH and CK-MB induced by DOX

Table 2 showed that DOX increased the activities of LDH and CK-MB when compared with healthy rats $(\mathrm{p}<0.05)$. There was no significant difference between DG and SG ( $p>0.05$ ), suggesting DMSO did not affect the activity of LDH and CK-MB. In contrast, sevoflurane and 3-MA reduced the activities of $\mathrm{LDH}$ and $\mathrm{CK}-\mathrm{MB}$ when compared with those from DG and SG $(p<0.05)$. All the results suggest that sevoflurane treatment is beneficial to reduce the activities of $\mathrm{LDH}$ and CK-MB in myocardial injury models.

\section{TUNEL assay of cellular apoptosis}

TUNEL analysis showed that the damaged DNA was stained brown. In CG, no obvious apoptotic cells were found. Compared with CG, cellular apoptosis was increased in DG and SG, and reached the highest level in LYG while the apoptosis was reduced in SevG and MG ( $\mathrm{p}<0.05$ ) (Fig. 2). The apoptosis indexes showed a similar trend at the cell level $(\mathrm{p}<0.05)($ Tables 3,4$)$.

\section{Protein concentration of $\mathrm{c} T \mathrm{~T} \mathrm{I}$ and apoptosis index}

For an animal experiment, serum concentration of cTnI was the lowest in CG among all groups. The level was increased in DG and SG, and reached the highest level in LYG while the levels were reduced in SevG and MG ( $\mathrm{p}<0.05)$ (Table 3). The apoptosis indexes showed the similar trend at the cell level $(\mathrm{p}<0.05)$ (Table 3 ). In cellular experiments, the concentration of cTnI showed similar results with those of animal experiments. The concentration of cTnI was the lowest in CG

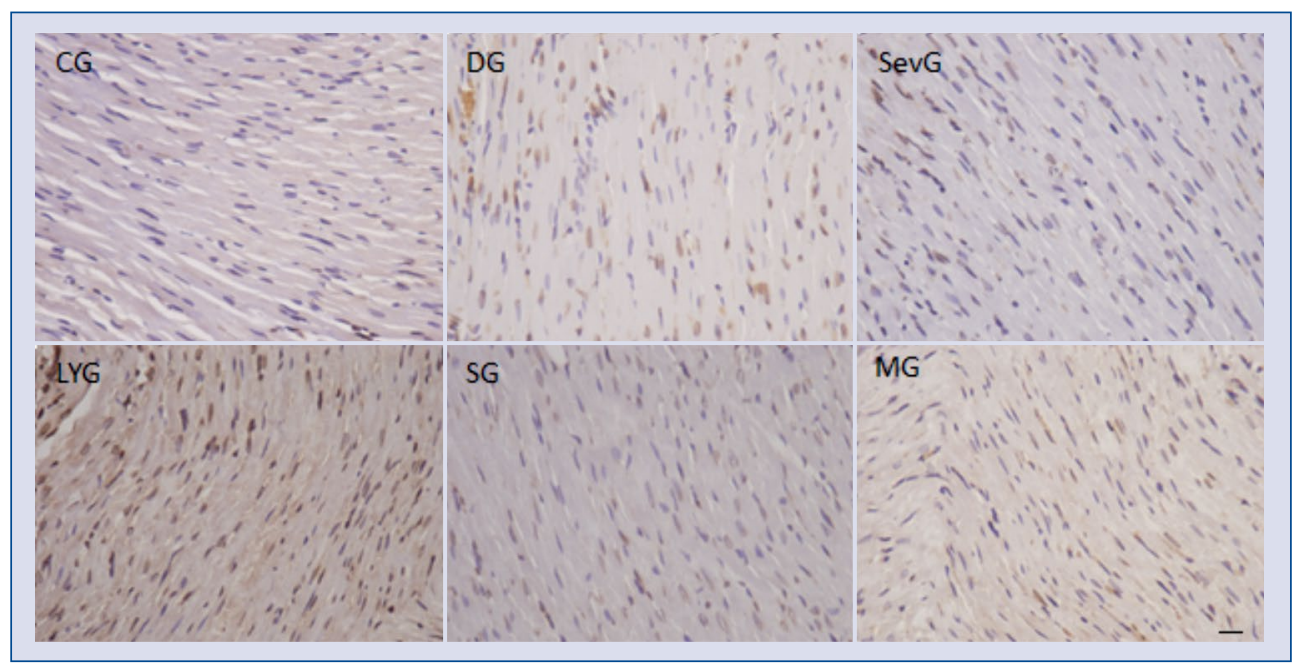

Figure 2. TUNEL analysis for myocardial cell apoptosis $(400 \times)$. Myocardial injury rat models were induced by doxorubicin and randomly divided into 5 groups according to different treatments: Doxorubicin group (DG), sevoflurane group (SevG), LY294002 group (Akt inhibitor, LYG), Solvent DMSO control group (SG) and autophagy inhibitor 3-methyladenine (3-MA) group (MG). Meanwhile, the healthy rats were assigned as a contro1 group (CG). In SevG, the rats were inhaled with $2.4 \%$ sevoflurane for $2 \mathrm{~h}$. In LYG, the rats were intraperitoneally injected with LY294002 $0.3 \mathrm{mg} / \mathrm{kg}$ injection in 200- $\mu \mathrm{L}$ DMSO. In SG, the rats were intraperitoneally injected with 200- $\mu \mathrm{L}$ DMSO. In MG, the rats were intraperitoneally injected with $\mathrm{mg} / \mathrm{kg} 3-\mathrm{MA}$ in 200- $\mu \mathrm{L}$ DMSO. 1 bar $=10 \mathrm{um}$. 
Table 3. The concentration of cardiac troponin I (cTnl) vand apoptosis rate of myocardial tissues of animal model.

\begin{tabular}{lcc}
\hline Groups & cTnl $[\mathrm{ng} / \mathrm{mL}]$ & Apoptosis [\%] \\
\hline CG & $0.21 \pm 0.03^{\#}$ & $7.71 \pm 2.01^{\#}$ \\
DG & $0.64 \pm 0.04^{*}$ & $37.9 \pm 2.82^{*}$ \\
SevG & $0.56 \pm 0.03^{*, \#}$ & $28.2 \pm 1.24^{*, \#}$ \\
LYG & $0.73 \pm 0.05^{*, \#}$ & $41.9 \pm 2.12^{*, \#}$ \\
SG & $0.60 \pm 0.04^{*}$ & $39.3 \pm 2.03^{*}$ \\
MG & $0.50 \pm 0.02^{*, \#}$ & $27.2 \pm 1.82^{*}$ \\
\hline
\end{tabular}

Myocardial injury rat models were induced by doxorubicin and randomly divided into five groups according to different treatments: Doxorubicin group (DG), sevoflurane group (SevG), LY294002 group (Akt inhibitor, LYG), Solvent DMSO control group (SG) and autophagy inhibitor 3-methyladenine (3-MA) group (MG). Meanwhile, the healthy rats were assigned as a control group (CG). In SevG, the rats were inhaled with $2.4 \%$ sevoflurane for $2 \mathrm{~h}$. In LYG, the rats were intraperitoneally injected with LY294002 $0.3 \mathrm{mg} / \mathrm{kg}$ injection in 200- $\mu$ L DMSO. In SG, the rats were intraperitoneally injected with 200- $\mu$ L DMSO. In MG, the rats were intraperitoneally injected with $30 \mathrm{mg} / \mathrm{kg} 3-\mathrm{MA}$ in $200-\mu \mathrm{L}$ DMSO.*p $<0.05$ via a CG group and \#p $<0.05$ via a DG group

Table 4. The concentration of cardiac troponin I (cTnl) and apoptosis rate of myocardial cells H9C2.

\begin{tabular}{lcc} 
Groups & cTnl $[\mathrm{ng} / \mathrm{mL}]$ & Apoptosis [\%] \\
\hline CG & $0.24 \pm 0.06^{\#}$ & $7.28 \pm 2.15^{\#}$ \\
DG & $0.56 \pm 0.07^{*}$ & $28.61 \pm 2.24^{*}$ \\
SevG & $0.42 \pm 0.04^{*, \#}$ & $19.42 \pm 1.82^{*, \#}$ \\
LYG & $0.65 \pm 0.07^{*, \#}$ & $32.23 \pm 2.61^{*, \#}$ \\
SG & $0.57 \pm 0.06^{*}$ & $27.12 \pm 1.93^{*}$ \\
MG & $0.44 \pm 0.05^{*, \#}$ & $17.82 \pm 2.03^{*, \#}$ \\
\hline
\end{tabular}

All the cells were divided into six groups ( $\mathrm{n}=6$ for each group): control group (CG), Doxorubicin group (DG), sevoflurane group (SevG), LY294002 group (LYG), Solvent DMSO control group (SG) and 3-methyladenine (3-MA) group (MG). In CG, the cells were not treated by doxorubicin. In DG, the model cells were incubated for $2 \mathrm{~h}$. In LYG, all the cells were treated LY294002 at a final concentration of $20 \mu \mathrm{M}$. In SevG, the cells were treated $2.4 \%$ sevoflurane for $2 \mathrm{~h}$. In SG, 1-mL cells were treated with 1- $\mu \mathrm{L}$ DMSO for $2 \mathrm{~h}$. In MG, 1 -mL cells were treated with $5 \mathrm{mM} 3-\mathrm{MA}$ in $1-\mu \mathrm{L}$ DMSO. ${ }^{*} \mathrm{p}<0.05$ via a CG group and \#p $<0.05$ via a DG group

among all groups. The level was increased in DG and SG, reaching the highest level in LYG while the levels were reduced in SevG and MG $(\mathrm{p}<0.05)$ (Table 4). The apoptosis indexes showed a similar trend at cell level as with the animal level $(\mathrm{p}<0.05)$ (Tables 3 and 4).

\section{p-Akt, p-mTOR and LC3-II levels}

For an animal experiment, the levels of p-Akt, p-mTOR were the highest in CG among all groups $(\mathrm{p}<0.05)$ (Fig. 3). The level was reduced in DG and SG and reached the lowest point in LYG while the levels were increased again in SevG and MG $(\mathrm{p}<0.05)$ (Fig. 3). Comparatively, the concentration of Akt and mTOR did not change significantly ( $p>0.05$ ). In contrast, the concentration of LC3-II showed the reverse changing trends among all groups. The level of LC3-II was lowest in CG among all groups $(\mathrm{p}<0.05)$ (Fig. 3). The level was increased in DG and SG and reached the highest point in LYG while the levels were reduced again in SevG and MG ( $<$ 0.05) (Fig. 3).

In the cellular experiment, similar results were also obtained. The levels of p-Akt, p-mTOR were highest in CG, among all groups $(\mathrm{p}<0.05)$ (Fig. 4). The level was reduced in DG and SG reaching the lowest point in LYG while the levels were increased again in SevG and MG $(\mathrm{p}<0.05)$ (Fig. 4). Comparatively, the concentration of Akt and mTOR did not change significantly ( $\mathrm{p}>0.05)$. In contrast, the concentration of LC3-II showed the reverse changing trend among all groups. The level of LC3-II was lowest in CG among all groups $(\mathrm{p}<0.05)$ (Fig. 4). The level was increased in DG and SG, reaching the highest point in LYG while the levels were reduced again in SevG and MG $(\mathrm{p}<0.05)$ (Fig. 4).

\section{Discussion}

In this study, a rat model of DOX-induced myocardial injury was established with higher activities of LDH and CK-MB when compared with healthy rats (Table 2). Myocardial apoptosis was increased with the higher level of serum cTnI in a rat model when compared with a healthy rat (Table 3 ). Ren et al. [15] reported that $2-\mu \mathrm{M}$ DOX can cause significant cardiac myocyte apoptosis in vitro. Therefore, $2-\mu \mathrm{M}$ DOX was added to cell culture in the present study. Conzen et al. [16] reported that $2.4 \%$ sevoflurane showed obvious therapeutic results in a rat model and thus the same concentration was used here. LY294002 and 3-MA was also administered based on previous literature [17].

Autophagy is a lysosomal degradation process involved in intracellular degradation of unwanted macromolecules. However, excessive autophagy will cause cellular energy depletion and internal environment disorder. To start cellular autophagic pathways, cell death is called programmed cell death type II [18], which unlike apoptosis (programmed cell death type I). Doxorubicin increases energy metabolism, intracellular calcium overload, and can induce autophagy and cardiotoxicity. Resveratrol has been reported to control autophagy by inhibiting S6K1 (p70 S6 kinase1), so as to reduce 


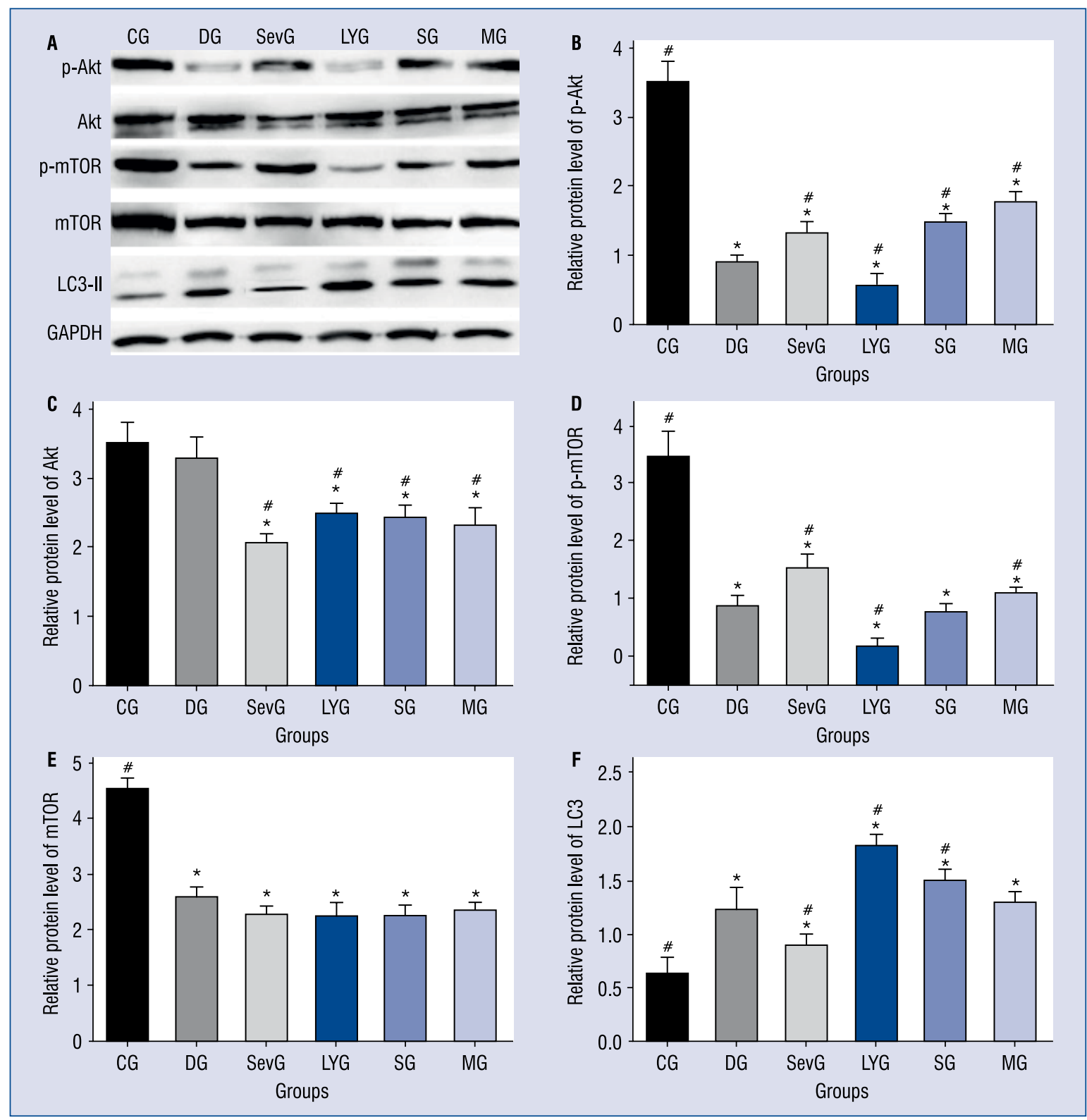

Figure 3. Western Blot analysis for the expression of Akt, p-Akt, mTOR, p-mTOR and cardiac troponin I in the rats with different treatment. Myocardial injury rat models were induced by doxorubicin and randomly divided into five groups according to different treatments: Doxorubicin group (DG), sevoflurane group (SevG), LY294002 group (Akt inhibitor, LYG), Solvent DMSO control group (SG) and autophagy inhibitor 3-methyladenine (3-MA) group (MG). Meanwhile, the healthy rats were assigned as a control group (CG). In SevG, the rats were inhaled with $2.4 \%$ sevoflurane for $2 \mathrm{~h}$. In LYG, the rats were intraperitoneally injected with LY294002 $0.3 \mathrm{mg} / \mathrm{kg}$ injection in $200-\mu \mathrm{L}$ DMSO. In SG, the rats were intraperi-toneally injected with 200- $\mu \mathrm{L}$ DMSO. In MG, the rats were intraperitoneally injected with $30 \mathrm{mg} / \mathrm{kg} 3-\mathrm{MA}$ in 200- $\mu$ L DMSO. * $p<0.05$ via a CG group and \#p < 0.05 via a DG group; GAPDH — glyceraldehyde 3-phosphate dehydrogenase.

DOX-induced cardiac cell death [19]. Present findings showed that the expression of LC3-II was increased in the model with DOX-induced cardiac injury and it significantly enhanced the level of serum cTnI, which suggests that autophagy activity was increased too. The application of autophagy inhibitor 3-MA reduced the levels of LC3-II and
cTnI (Figs. 3, 4, Tables 3, 4) to inhibit autophagy, which implied that DOX reduced autophagy damage for cardiomyocytes.

Autophagy is regulated by autophagy-related genes (Atg), which comprises: Atg1 kinase complex (Atg1-mAtg13-FIP200-Atg101), starts the activity of autophagy; PI3K kinase complex type III 


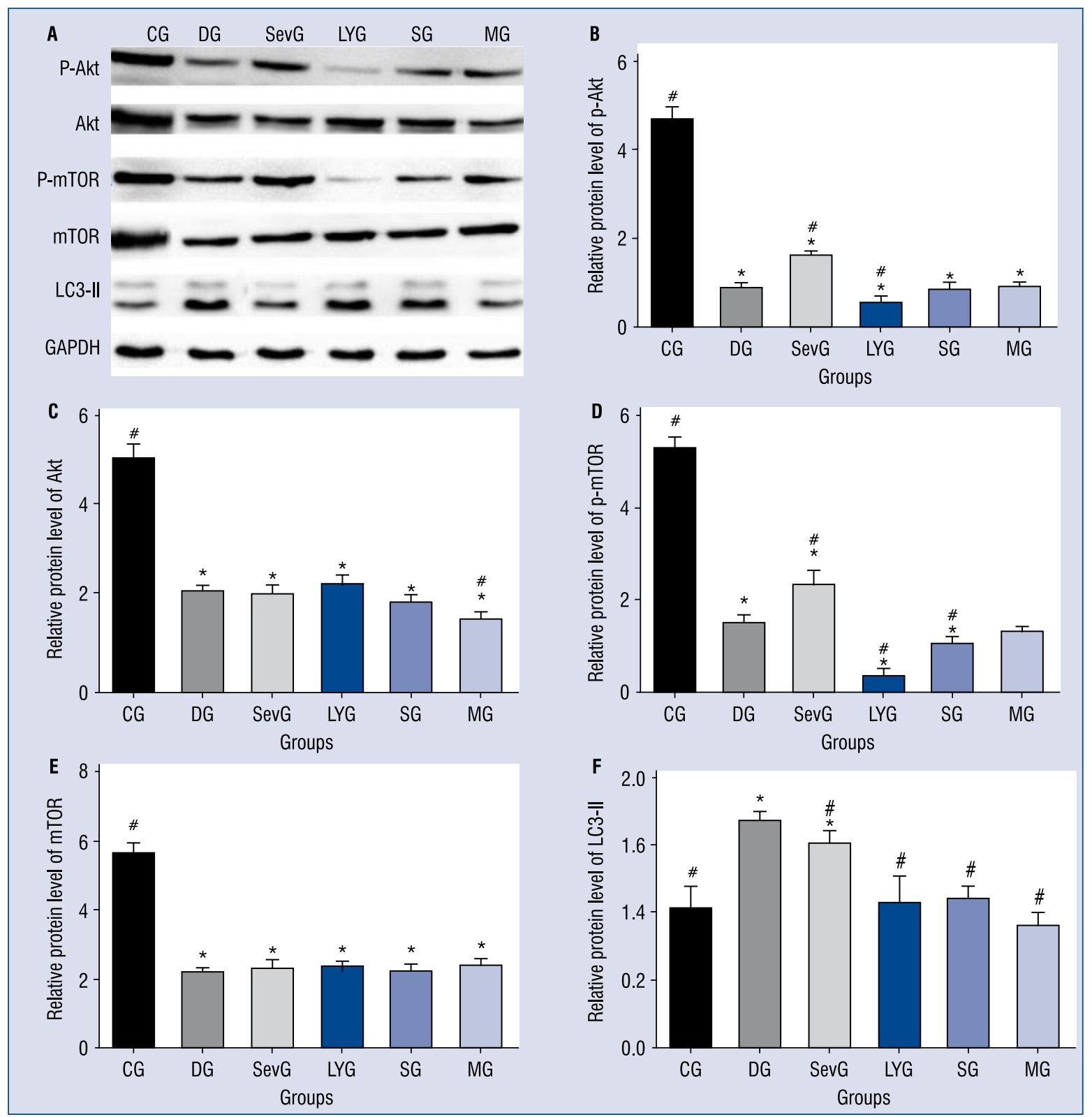

Figure 4. Western Blot analysis for the expression of Akt, p-Akt, mTOR, p-mTOR and cardiac troponin I in myocardial cells $\mathrm{H} 9 \mathrm{c} 2$ with different treatment. All the cells were assigned into six groups according to different treatment: control group (CG), Doxorubicin group (DG), sevoflurane group (SevG), LY294002 group (LYG), Solvent DMSO control group (SG) and autophagy inhibitor 3-methyladenine (3-MA) group (MG). In CG, the cells were not treated by doxorubicin. In DG, the model cells were incubated for $2 \mathrm{~h}$. In LYG, all the cells were treated LY294002 at a final concentration of $20 \mu \mathrm{M}$. In SevG, the cells were treated $2.4 \%$ sevoflurane for $2 \mathrm{~h}$. In $\mathrm{SG}$, the cells were treated with equal volume of DMSO for $2 \mathrm{~h}$. In MG, the cells were treated with 3-MA at a final concentration of $5 \mathrm{mM} .{ }^{*} \mathrm{p}<0.05$ via a CG group and $\# p<0.05$ via a DG group; GAPDH - glyceraldehyde 3-phosphate dehydrogenase.

(Vps34-PI3KIII-Becline1), promotes the formation of phagosome [20]. Doxorubicin can damage mitochondria, endoplasmic reticulum and leads to adenosine triphosphate production. Dysfunction of protein synthesis is often caused by myocardial nutritional deprivation, which is also a typical cause of autophagy. In the process, the main target protein is involved in astrocyte-defined medium (ADM) regulation signaling pathway, which induces autophagy. Under normal circumstances, mTOR kinase complex binds Atg1, thereby inhibiting autophagy. When lack of nutrition is prominent, mTOR kinase will be dissociated with Atg1, and Atg1 is dephosphorylated. Therefore, Atg1 kinase 
complex and autophagy is activated [21]. PI3K/Akt pathway is a major upstream regulator of $\mathrm{mTOR}$ signaling pathway, and PI3K/Akt/mTOR signaling pathway also regulates cell growth. Present findings showed that the levels of phosphorylated Akt and mTOR were highest in CG and lowest in DG and LYG while sevoflurane improved the phosphorylated states of Akt and mTOR in PI3K/Akt/mTOR signaling pathway (Fig. 3B, D, and Fig. 4B, D). Thus, sevoflurane may activate the activity of PI3K/Akt/ /mTOR signaling pathway by affecting the phosphorylated sates of main proteins in the pathway.

Clinical trials confirmed that sevoflurane had positive cardioprotective effect during bypass surgery of coronary artery [22]; Sevoflurane is an excellent adjuvant to propofol for protecting the heart in patients with coronary heart disease. A previous study found that sevoflurane might induce anti-apoptotic protein Bcl-2, inhibited Bax and Caspase [23] and reduced DOX-induced injury. Inamura et al. [24] demonstrated that sevoflurane treatment reduced ischemia-reperfusion apoptosis. Zhang et al. [25] reported sevoflurane treatment inhibited autophagy and reduced myocardial ischemia and reperfusion injury by affecting PI3K/ /Akt signaling pathway.

Present findings showed that sevoflurane treatment reduced cardiac injury by inhibiting the expression of LC3-II and increasing the level of phosphorylated protein p-Akt and p-mTOR (Figs. 3,4 ), which is closely associated PI3K/Akt/mTOR pathway. The results suggest that the decrease of p-Akt level promotes autophagy, resulting in myocardial damage. Doxorubicin induced the inhibition of phosphorylation of Akt and mTOR. Further application of PI3K inhibitor LY294002 also further inhibited the phosphorylation of Akt and mTOR, and increased the expression of LC3-II. Meanwhile, sevoflurane showed protective functions for myocardial injury by activating the phosphorylation of PI3K/Akt/mTOR pathway and inhibiting the expression of cTnI.

\section{Limitations of the study}

There were some limitations for present work: 1) The small sample size was the main limitation of present study because the number of rats was restricted by the Animal Ethical Committee; 2) the relationship between phosphorylated Akt and $\mathrm{mTOR}$, and cardinal injury biomarker cTnI and or autophagy factor LC3-II was not confirmed, although the changing trend was consistent; 3 ) 3 rats had ascites and 4 rats died. The number was too low to be analyzed by using a statistical method. These rats were removed from the final statistical analysis. The reasons for those rats having ascites and dying can be complex. Two rats which died were from LYG, and thus DOX and LY294002 may have a synergistic function for causing myocardial injury. Cell death was reported when PI3K/Akt pathway was blocked by the PI3K inhibitor LY294002 [1]. The optimization is going to be performed in future, including one-time dosage, interval time and accumulated dosages.

\section{Conclusions}

In sum, sevoflurane treatment reduces myocardial cell autophagy by affecting the phosphorylated sates of main proteins of PI3K/Akt/mTOR signaling pathway, and inhibiting the expression of cTnI. The exact mechanism of DOX-induced myocardial damage is not yet clear. The critical role of autophagy has been gradually recognized. Given the important role of phosphorylation of Akt and mTOR for myocardial injury, potential drugs should be developed for myocardial protection.

\section{Acknowledgements}

We are very grateful to all three anonymous reviewers for their critical and strategic comments, which has certainly improved the quality of present paper. The work was supported by the Key projects of Zhejiang medical and health platform (No.2014ZDA031), the Lishui high level talent training project (No.2013RC04) and the Lishui Key Discipline Project (No.2014ZDxk06).

\section{Conflict of interest: None declared}

\section{References}

1. Prysyazhna O, Burgoyne JR, Scotcher J, et al. Phosphodiesterase 5 Inhibition Limits Doxorubicin-induced Heart Failure by Attenuating Protein Kinase G I $\alpha$ Oxidation. J Biol Chem. 2016; 291(33): 17427-17436, doi: 10.1074/jbc.M116.724070, indexed in Pubmed: 27342776.

2. Sato M, Yamanaka H, Iwasaki M, et al. Altered Phosphatidylinositol 3-Kinase and Calcium Signaling in Cardiac Dysfunction After Brain Death in Rats. Ann Thorac Surg. 2016; 102(2): 556-563, doi: 10.1016/j.athoracsur.2016.02.014, indexed in Pubmed: 27130251.

3. Shiojima I, Walsh K. Regulation of cardiac growth and coronary angiogenesis by the Akt/PKB signaling pathway. Genes Dev. 2006; 20(24): 3347-3365, doi: 10.1101/gad.1492806, indexed in Pubmed: 17182864.

4. Cao J, Xie H, Sun Y, et al. Sevoflurane post-conditioning reduces rat myocardial ischemia reperfusion injury through an increase in NOS and a decrease in phopshorylated NHE1 levels. Int J Mol Med. 2015; 36(6): 1529-1537, doi: 10.3892/ijmm.2015.2366, indexed in Pubmed: 26459736. 
5. Sahu BD, Kumar JM, Kuncha M, et al. Baicalein alleviates doxorubicin-induced cardiotoxicity via suppression of myocardial oxidative stress and apoptosis in mice. Life Sci. 2016; 144: 8-18, doi: 10.1016/j.lfs.2015.11.018, indexed in Pubmed: 26606860.

6. Bartlett JJ, Trivedi PC, Yeung P, et al. Doxorubicin impairs cardiomyocyte viability by suppressing transcription factor EB expression and disrupting autophagy. Biochem J. 2016; 473(21): 37693789, doi: 10.1042/BCJ20160385, indexed in Pubmed: 27487838.

7. Li S, Liu C, Gu L, et al. Autophagy protects cardiomyocytes from the myocardial ischaemia-reperfusion injury through the clearance of CLP36. Open Biol. 2016; 6(8), doi: 10.1098/rsob.160177, indexed in Pubmed: 27512143.

8. Xia Q, Zheng Yi, Jiang W, et al. Valproic acid induces autophagy by suppressing the Akt/mTOR pathway in human prostate cancer cells. Oncol Lett. 2016; 12(3): 1826-1832, doi: 10.3892/ ol.2016.4880, indexed in Pubmed: 27588130.

9. Ma R, Wang X, Peng P, et al. $\alpha$-Lipoic acid inhibits sevoflurane-induced neuronal apoptosis through PI3K/Akt signalling pathway. Cell Biochem Funct. 2016; 34(1): 42-47, doi: 10.1002/cbf.3163, indexed in Pubmed: 26781804.

10. Jacinto E, Facchinetti V, Liu D, et al. SIN1/MIP1 maintains rictormTOR complex integrity and regulates Akt phosphorylation and substrate specificity. Cell. 2006; 127(1): 125-137, doi: 10.1016/j. cell.2006.08.033, indexed in Pubmed: 16962653.

11. Dimmeler S, Assmus B, Hermann C, et al. Fluid shear stress stimulates phosphorylation of Akt in human endothelial cells: involvement in suppression of apoptosis. Circ Res. 1998; 83(3): 334-341, doi: 10.1161/01.res.83.3.334, indexed in Pubmed: 9710127.

12. Altomare DA, Wang HQ, Skele KL, et al. AKT and mTOR phosphorylation is frequently detected in ovarian cancer and can be targeted to disrupt ovarian tumor cell growth. Oncogene. 2004; 23(34): 5853-5857, doi: 10.1038/sj.onc.1207721, indexed in Pubmed: 15208673.

13. Kim K, Park C, Kwon D, et al. Silicon nanowire biosensors for detection of cardiac troponin I (cTnI) with high sensitivity. Biosens Bioelectron. 2016; 77: 695-701, doi: 10.1016/j. bios.2015.10.008, indexed in Pubmed: 26496224.

14. Romano MM, Pazin-Filho A, O'Connel JL, et al. Early detection of doxorubicin myocardial injury by ultrasonic tissue characterization in an experimental animal model. Cardiovasc Ultrasound. 2012; 10: 40, doi: 10.1186/1476-7120-10-40, indexed in Pubmed: 23046747.

15. Ren D, Zhu Q, Li J, et al. Overexpression of angiopoietin-1 reduces doxorubicin-induced apoptosis in cardiomyocytes. J Biomed
Res. 2012; 26(6): 432-438, doi: 10.7555/JBR.26.20120006, indexed in Pubmed: 23554782.

16. Conzen PF, Vollmar B, Habazettl H, et al. Systemic and regional hemodynamics of isoflurane and sevoflurane in rats. Anesth Analg. 1992; 74(1): 79-88, doi: 10.1213/00000539-19920100000014, indexed in Pubmed: 1734802.

17. Kim DE, Kim B, Shin HS, et al. The protective effect of hispidin against hydrogen peroxide-induced apoptosis in H9c2 cardiomyoblast cells through Akt/GSK-3 $\beta$ and ERK1/2 signaling pathway. Exp Cell Res. 2014; 327(2): 264-275, doi: 10.1016/j. yexcr.2014.07.037, indexed in Pubmed: 25128810.

18. Kang C, Avery L. To be or not to be, the level of autophagy is the question: dual roles of autophagy in the survival response to starvation. Autophagy. 2008; 4(1): 82-84, doi: 10.4161/auto.5154, indexed in Pubmed: 17952023.

19. Xu X, Chen K, Kobayashi S, et al. Resveratrol attenuates doxorubicin-induced cardiomyocyte death via inhibition of $\mathrm{p} 70$ S6 kinase 1-mediated autophagy. J Pharmacol Exp Ther. 2012; 341(1): 183-195, doi: 10.1124/jpet.111.189589, indexed in Pubmed: 22209892.

20. Jung $\mathrm{CH}$, Ro $\mathrm{SH}$, Cao J, et al. mTOR regulation of autophagy. FEBS Lett. 2010; 584(7): 1287-1295, doi: 10.1016/j.febslet.2010.01.017, indexed in Pubmed: 20083114.

21. Papinski D, Kraft C. Regulation of Autophagy By Signaling Through the Atg1/ULK1 Complex. J Mol Biol. 2016; 428(9 Pt A): $1725-1741$, doi: $10.1016 /$ j.jmb.2016.03.030, indexed in Pubmed: 27059781.

22. Ceyhan D, Tanriverdi B, Bilir A. Comparison of the effects of sevoflurane and isoflurane on myocardial protection in coronary bypass surgery. Anadolu Kardiyol Derg. 2011; 11(3): 257-262, doi: 10.5152/akd.2011.059, indexed in Pubmed: 21466991.

23. Wang Y, Zuo M. Nicotinamide improves sevoflurane-induced cognitive impairment through suppression of inflammation and antiapoptosis in rat. Int J Clin Exp Med. 2015; 8(11): 20079-20085, indexed in Pubmed: 26884920.

24. Inamura $\mathrm{Y}$, Miyamae M, Sugioka S, et al. Sevoflurane postconditioning prevents activation of caspase 3 and 9 through antiapoptotic signaling after myocardial ischemia-reperfusion. J Anesth. 2010; 24(2): 215-224, doi: 10.1007/s00540-010-0877-6, indexed in Pubmed: 20177944.

25. Zhang J, Wang C, Yu S, et al. Sevoflurane postconditioning protects rat hearts against ischemia-reperfusion injury via the activation of PI3K/AKT/mTOR signaling. Sci Rep. 2014; 4: 7317, doi: 10.1038/srep07317, indexed in Pubmed: 25471136. 